\title{
Uniquely cycle-saturated graphs
}

\author{
Paul S. Wenger* and Douglas B. West ${ }^{\dagger}$
}

August 25, 2021

\begin{abstract}
Given a graph $F$, a graph $G$ is uniquely $F$-saturated if $F$ is not a subgraph of $G$ and adding any edge of the complement to $G$ completes exactly one copy of $F$. In this paper we study uniquely $C_{t}$-saturated graphs. We prove the following: (1) a graph is uniquely $C_{5}$-saturated if and only if it is a friendship graph. (2) There are no uniquely $C_{6}$-saturated graphs or uniquely $C_{7}$-saturated graphs. (3) For $t \geq 6$, there are only finitely many uniquely $C_{t}$-saturated graphs (we conjecture that in fact there are none).
\end{abstract}

Keywords: 05C35; saturation; unique saturation

\section{Introduction}

Given a graph $F$, a graph $G$ is $F$-saturated if $F$ is not a subgraph of $G$ but is a subgraph of $G+e$ for every edge $e$ in the complement $\bar{G}$ of $G$. In 1907, Mantel [7] proved that the $n$-vertex $K_{3}$-saturated graph with the most edges is $K_{[n / 2\rceil,\lfloor n / 2]}$. Turán [8] generalized this result, proving that the $n$-vertex $K_{t}$-saturated graph with the most edges is the complete $(t-1)$-partite graph with partite sets as balanced as possible. Erdős, Hajnal, and Moon [4] proved that the $n$-vertex $K_{t}$-saturated graph with the fewest edges is $K_{t-2} \triangleleft \bar{K}_{n-t+2}$, where the join $G \bowtie H$ of graphs $G$ and $H$ consists of the disjoint union of $G$ and $H$ plus edges connecting all vertices of $G$ to all vertices of $H$.

There is an important distinction between $K_{t}$-saturated graphs with the most and the fewest edges. When an edge is added to a largest $n$-vertex $K_{t}$-saturated graph, roughly $\left(\frac{n}{t-1}\right)^{t-2}$ copies of $K_{t}$ are formed. In contrast, when an edge is added to a smallest $n$-vertex $K_{t}$-saturated graph, exactly one copy of $K_{t}$ is formed. Given a graph $F$ and an $F$-saturated

\footnotetext{
*School of Mathematical Sciences, Rochester Institute of Technology, Rochester, NY; pswsma@rit.edu.

${ }^{\dagger}$ Departments of Mathematics, Zhejiang Normal University, China, and University of Illinois, USA , west@math. uiuc.edu. Research supported by Recruitment Program of Foreign Experts, 1000 Talent Plan, State Administration of Foreign Experts Affairs, China
} 
graph $G$, we say that $G$ is uniquely $F$-saturated if the addition of any edge to $G$ completes exactly one copy of $F$.

Questions about uniquely $F$-saturated graphs focus on their existence. Cooper, LeSaulnier, Lenz, Wenger, and West [3] initiated the study of uniquely $F$-saturated graphs by determining all uniquely $C_{4}$-saturated graphs, where $C_{t}$ denotes the $t$-vertex cycle; there are exactly 10 such graphs. They also observed that a graph is uniquely $C_{3}$-saturated if and only if it is a star or a Moore graph of diameter 2 .

Stars have a dominating vertex, but Moore graphs of diameter 2 do not. If $G$ is uniquely $K_{t}$-saturated, then $K_{m} \otimes G$ is uniquely $K_{m+t}$-saturated and has dominating vertices. Cooper 2] conjectured that for $t \geq 2$, only finitely many $K_{t}$-saturated graphs have no dominating vertices. Hartke and Stolee [6] computationally found new examples for small $t$ of $K_{t}$-saturated graphs without dominating vertices and found two constructions of $K_{t}$-saturated graphs without dominating vertices based on Cayley graphs, each valid for infinitely many $t$.

Berman, Chappell, Faudree, Gimble, and Hartman [1] studied uniquely tree-saturated graphs. They proved if $T$ is a tree, then there exist infinitely many uniquely $T$-saturated graphs if and only if $T$ is a balanced double star.

When $F$ has $t$ vertices, every complete graph with fewer than $t$ vertices trivially is uniquely $F$-saturated, since there are no edges to consider adding. Let a uniquely $C_{t^{-}}$ saturated graph be nontrivial if it has at least $t$ vertices. In Section 2 we establish structural lemmas about such graphs. In Section 3 we prove that the nontrivial uniquely $C_{5}$-saturated graphs are precisely the graphs consisting of edge-disjoint triangles with one common vertex (adjacent to all others). Such graphs are also called friendship graphs, because they are the graphs in which every two vertices have exactly one common neighbor (proved initially by Erdős, Rényi, and Sós [5] and later reproved by others). In Section 4 we prove that there are no nontrivial uniquely $C_{6}$-saturated graphs or uniquely $C_{7}$-saturated graphs. Finally, in Section 5 we prove the following theorem.

Theorem 1.1. For $t \geq 6$, there are finitely many uniquely $C_{t}$-saturated graphs.

In light of our results, we make the following conjecture.

Conjecture 1.2. For $t \geq 6$ there are no nontrivial uniquely $C_{t}$-saturated graphs.

We have verified Conjecture 1.2 for $t=8$, but the proof is quite long and does not contain any new ideas beyond those used in the proofs of Theorems 4.1 and 4.2, thus we do not include the proof here. 


\section{Structural lemmas}

In keeping with the convention of using $k$-cycle for a copy of $C_{k}$, we refer to a path with $k$ vertices as a $k$-path. We use $\left\langle v_{1}, \ldots, v_{k}\right\rangle$ to denote the $k$-path with vertices $v_{1}, \ldots, v_{k}$ indexed in order. We use $\left[v_{1}, \ldots, v_{k}\right]$ to denote the $k$-cycle with vertices $v_{1}, \ldots, v_{k}$ indexed in order. For vertices $x$ and $y$ in a graph $G$, we use $d_{G}(x, y)$ to denote the distance between $x$ and $y$ and $d_{G}(x)$ for the degree of $x$.

We begin with an elementary observation about uniquely $C_{t}$-saturated graphs.

Observation 2.1. Any two vertices in a uniquely $C_{t}$-saturated graph are the endpoints of at most one $t$-path, and such a path exists if and only if they are not adjacent.

A block in a graph is a maximal subgraph not having a cut-vertex. Thus it is a maximal 2-connected subgraph or has a single edge that is a cut-edge.

Lemma 2.2. Every block in a uniquely $C_{t}$-saturated graph is uniquely $C_{t}$-saturated. In particular, blocks with fewer than $t$ vertices are complete graphs.

Proof. Let $x$ and $y$ be nonadjacent vertices in a non-complete block $B$ of such a graph $G$. Since $B$ is a maximal 2-connected subgraph, the unique $t$-path in $G$ with endpoints $x$ and $y$ is contained in $B$. Thus $|V(B)| \geq t$, and $B$ is uniquely $C_{t^{-}}$saturated.

We next bound the size of complete blocks in nontrivial uniquely $C_{t}$-saturated graphs.

Lemma 2.3. Every complete block in a nontrivial uniquely $C_{t}$-saturated graph has at most three vertices.

Proof. The claim is trivial for $t=3$, so assume $t \geq 4$. Let $G$ be a non-complete graph. Let $B$ and $B^{\prime}$ be blocks in $G$, with a common vertex $v$, such that $B$ is a complete graph and has at least four vertices. Let $u$ and $x$ be vertices other than $v$ in $B$ and $B^{\prime}$, respectively. The vertices $u$ and $x$ are nonadjacent, and every $t$-path $P$ with endpoints $u$ and $x$ contains $v$.

If $P$ is unique, then the portion of $P$ in $B$ must have length 1 , since $B$ has at least four vertices. Since $t \geq 4$, this implies that $P$ has at least two edges in $B^{\prime}$. Hence the neighbor $x^{\prime}$ of $x$ on $P$ is not $v$. Now $x^{\prime}$ and $u$ are not adjacent, but there are multiple 3 -paths in $B$ with endpoints $u$ and $v$, so $G$ is not uniquely $C_{t}$-saturated.

Using Lemma 2.3, we can restrict our attention to 2-connected graphs when $t \geq 6$.

Lemma 2.4. If $t \geq 6$, then every nontrivial uniquely $C_{t}$-saturated graph contains a block that is not a complete graph. In fact, no two blocks with a common vertex are complete. 
Proof. Let $G$ be a nontrivial uniquely $C_{t}$-saturated graph. By Lemma 2.3, the union of two complete blocks with a common vertex $v$ has at most five vertices. Hence if $u$ and $x$ are vertices of these blocks other than $v$, then $u$ and $x$ are nonadjacent but are not the endpoints of a $t$-path. The contradiction implies that no two neighboring blocks can be complete.

For $t \geq 6$, Lemma 2.4 reduces Conjecture 1.2 to the consideration of 2-connected graphs.

Corollary 2.5. For $t \geq 6$, if there are no 2-connected nontrivial uniquely $C_{t}$-saturated graphs, then there are no nontrivial uniquely $C_{t}$-saturated graphs.

We next forbid certain subgraphs, aiming to forbid certain cycle lengths. Let $H_{m, \ell}$ be the graph that consists of a $2 m$-cycle with a pendant path of length $\ell$ (see Figure 1).

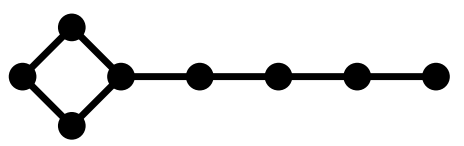

$H_{2,4}$

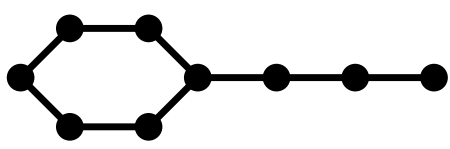

$H_{3,3}$

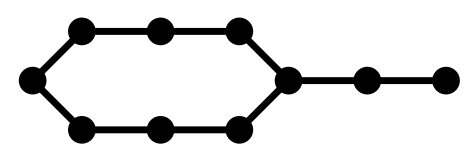

$H_{4,2}$

Figure 1: Forbidden subgraphs for uniquely $C_{7}$-saturated graphs.

Lemma 2.6. If $k<t$ with $t \geq 3$, then no uniquely $C_{t}$-saturated graph contains $H_{k, t-k-1}$.

Proof. The diameter of $H_{k, t-k-1}$ is $t-1$, and there are two $t$-paths connecting two vertices at distance $t-1$.

Lemma 2.7. For $t \geq 5$, a uniquely $C_{t}$-saturated graph $G$ cannot contain $C_{2 t-2}$ or $C_{2 t-4}$.

Proof. Note that $C_{2 t-2}=H_{t-1,0}$, so Lemma 2.6 applies. If $G$ contains $C_{2 t-4}$, then avoiding $H_{t-2,1}$ requires $|V(G)|=2 t-4$. Let $C$ be a spanning cycle in $G$, with $C=\left[v_{0}, \ldots, v_{2 t-5}\right]$ (indices taken modulo $2 t-4$ ). If $G$ contains a chord of $G$, then it creates cycles of lengths $l$ and $2 t-2-l$, for some $l$. If $l=2 k$, then $H_{k, t-k-1} \subseteq G$.

If $l=2 k+1$ is odd, then we may assume by symmetry that the chord is $v_{k} v_{-k}$. Now $G$ contains two $t$-paths with endpoints $v_{0}$ and $v_{t-2}$, using the chord in opposite directions.

Hence $G=C_{2 t-4}$, but now opposite vertices are not connected by any $t$-path.

The girth of a graph is the minimum length of a cycle in it.

Lemma 2.8. For $t \geq 5$, a uniquely $C_{t}$-saturated graph $G$ has girth at most $t+1$. 
Proof. Let $x$ and $y$ be two vertices in $G$ such that $d_{G}(x, y)=2$, and let $z$ be a common neighbor of $x$ and $y$. Let $P$ be the unique $t$-path with endpoints $x$ and $y$. If $P$ does not contain $z$, then the union of $P$ and the path $\langle x, z, y\rangle$ is a $(t+1)$-cycle. If $P$ contains $z$, then the union of $P$ and $\langle x, z, y\rangle$ contains a cycle with length at most $t$.

Two vertices having the same neighborhood are twins.

Lemma 2.9. For $t \geq 4$, a uniquely $C_{t}$-saturated graph cannot contain twins.

Proof. Let $x$ and $y$ be twins in a graph $G$; note that twins are nonadjacent. If $d(x)=$ $d(y)=1$, then the only cycle completed by added $x y$ is a 3-cycle, so $G$ is not uniquely $C_{t}$-saturated. If $d(x)=d(y) \geq 2$ and there is a $t$-path $P$ with endpoints $x$ and $y$, then let $x^{\prime}$ and $y^{\prime}$ be the neighbors of $x$ and $y$ on $P$, respectively. Because $x$ and $y$ are twins, $x^{\prime} y, x y^{\prime} \in E(G)$. Reversing the central $(t-2)$-path of $P$ yields a second $t$-path with endpoints $x$ and $y$ containing the edges $x y^{\prime}$ and $x^{\prime} y$. Thus $G$ is not uniquely $C_{t}$-saturated.

A chordal path of a cycle $C$ is a path of length at least 2 whose endpoints are in $C$ and whose internal vertices are not in $C$.

Lemma 2.10. For $t \geq 6$, every nontrivial uniquely $C_{t}$-saturated graph $G$ contains an even cycle of length at most $2 t-6$.

Proof. By Lemma 2.8, $G$ has girth at most $t+1$. By Lemma 2.7, $G$ does not contain $C_{2 t-2}$ or $C_{2 t-4}$. Since $t+2 \leq 2 t-2$, we may assume that the girth of $G$ is odd and that $G$ has no even cycle of length at most $2 t-2$.

First suppose that $G$ contains a cycle $C$ of length $2 k+1$ such that $2 \leq k \leq\lfloor t / 2\rfloor$. The prohibition of short even cycles implies that $C$ has no chord. Let $x$ and $y$ be nonconsecutive vertices in $C$ such that $d_{C}(x, y) \neq t-1$; thus $x$ and $y$ are not adjacent. A $t$-path with endpoints $x$ and $y$ contains a chordal path of $C$ with length at most $t-1$. Combining this chordal path with a path from $x$ to $y$ along $C$ yields an even cycle with length at most $2 t-2$ in $G$, a contradiction.

Now suppose that $G$ contains a 3-cycle but no cycle of length $2 k+1$ with $2 \leq k \leq\lfloor t / 2\rfloor$. Let $[x, y, z]$ be a 3 -cycle $C$, and let $x$ be a vertex of $C$ having a neighbor $x^{\prime} \notin V(C)$. Since by assumption $G$ has no 4 -cycle, $x^{\prime} y \notin E(G)$. Hence $G$ has a $t$-path $P$ with endpoints $x^{\prime}$ and $y$. Now $P$ contains one of the following: a subpath of length at least 3 with endpoints $x^{\prime}$ and $x$, a chordal path of length at least 2 connecting two vertices in $V(C)$, or a path connecting $x^{\prime}$ and a vertex in $\{y, z\}$ with all internal vertices outside $C$. In all cases, $G$ contains an even cycle of length at most $t+2$ or an odd cycle of length $2 k+1$ with $2 \leq k \leq\lfloor t / 2\rfloor$. 
In light of Lemma 2.10 guaranteeing an even cycle of length at most $2 t-6$, an approach to proving Conjecture 1.2 that there is no uniquely $C_{t}$-saturated graph for $t \geq 6$ is to prove that such a graph has no such even cycle. Although we cannot completely exclude $(2 t-6)$-cycles for all $t$, we can greatly restrict the graphs that contain them. We state a general lemma without proof, because we present the proof of Conjecture 1.2 only through $t=7$. The ad hoc proof excluding 8-cycles when $t=7$ is shorter than the general proof of this lemma.

Lemma 2.11. For $t \geq 7$, if a nontrivial uniquely $C_{t}$-saturated graph $G$ contains a $(2 t-6)$ cycle $C$ and $R=V(G)-V(C)$, then (1) $G[R]$ has no edges, (2) every vertex of $R$ has exactly two neighbors on $C$, separated by odd distance (at least 3) along $C$, and (3) all vertices of $R$ have the same distance along $C$ between their neighbors on $C$. Also, (4) all chords of $C$ join vertices at even distance along $C$.

\section{Uniquely $C_{5}$-saturated graphs}

As mentioned in the introduction, the Friendship Theorem of Erdős, Rényi, and Sós [5] states that if every two vertices in a graph have exactly one common neighbor, then some vertex is adjacent to all others. As they noted, this immediately implies that the graph consists of edge-disjoint triangle with one common vertex.

In such a graph, there is only one type of missing edge, joining two of the triangles. Adding this to two edges from each of the two triangles completes a unique 5-cycle. Hence friendship graphs are uniquely $C_{5}$-saturated.

Theorem 3.1. A graph is a nontrivial uniquely $C_{5}$-saturated graph if and only if it is a friendship graph with at least five vertices.

Proof. We have noted that the condition is sufficient. For the converse, let $G$ be a nontrivial uniquely $C_{5}$-saturated graph. Our proof depends on five graphs that cannot be subgraphs of $G$. Already $H_{2,2}$ and $H_{3,1}$ are excluded by Lemma 2.6. Let $F$ consist of $K_{4}$ plus a pendant edge at one vertex, and let $F^{\prime}$ consist of the 5-vertex friendship graph plus a pendant edge at a vertex of degree 2. Figure 2 illustrates that $F, F^{\prime}$, and the complete bipartite graph $K_{2,3}$ are all forbidden as subgraphs of $G$, since each has a nonadjacent vertex pair that when added completes at least two 5-cycles.

By Lemma 2.8, $G$ has girth at most 6; by Lemma 2.7, $G$ has no 6-cycle. By definition, $G$ has no 5-cycle.

Suppose first that $G$ has a 4 -cycle; let $S$ be its vertex set. Let $R=V(G)-S$. Because $G$ is connected and $H_{2,2} \nsubseteq G$, each vertex in $R$ has a neighbor in $S$, and $R$ is an independent 

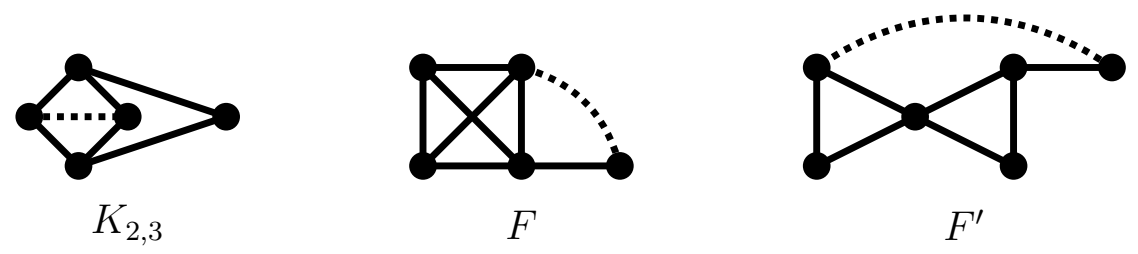

Figure 2: Three graphs forbidden as subgraphs of uniquely $C_{5}$-saturated graphs.

set. Because $C_{5}, K_{2,3} \nsubseteq \subseteq G$, each vertex in $R$ has exactly one neighbor in $S$. Therefore $S$ is the vertex set of a block in $G$, and by Lemma $2.2 S$ is a clique. Since $F \nsubseteq G$, we conclude that $R=\emptyset$ and $G=K_{4}$. Thus no nontrivial uniquely $C_{5}$-saturated graph has a 4-cycle.

We conclude that $G$ has no 4 -cycle but has a 3 -cycle, say $[x, y, z]$. Since $G$ is nontrivial and connected, we may assume by symmetry that $x$ has a neighbor $x^{\prime}$ not in $\{y, z\}$. Since $G$ has no 4-cycle, $y$ and $z$ are not adjacent to $x^{\prime}$. Since $G$ has no 4-cycle or 6-cycle, the unique 5-path $P$ with endpoints $x^{\prime}$ and $y$ contains $x$. It must be $\left\langle x^{\prime}, w, x, z, y\right\rangle$, where $w$ is a common neighbor of $x^{\prime}$ and $x$. Since $F^{\prime} \nsubseteq G$, we conclude that $y, z, x^{\prime}$, and $w$ have no other neighbors in $G$. Repeating the argument shows that $x$ is a dominating vertex and $G-x$ is a disjoint union of copies of $K_{2}$, so $G$ is a friendship graph with at least five vertices.

The case in Theorem 3.1 where $G$ has no 4 -cycle shows why the proof of Lemma 2.10 is not valid for $t=5$. The common neighbor of $x^{\prime}$ and $x$ yields the 5-path with endpoints $x^{\prime}$ and $y$ without creating a 4-cycle.

\section{Uniquely $C_{6}$ - and $C_{7}$-saturated graphs}

In this section, we prove that there are no nontrivial uniquely $C_{6}$-saturated or uniquely $C_{7}$-saturated graphs. Our proofs depend on successively forbidding cycles of various lengths.

Theorem 4.1. There are no nontrivial uniquely $C_{6}$-saturated graphs.

Proof. Let $G$ be a uniquely $C_{6}$-saturated graph. By Corollary 2.5 , we may assume that $G$ is 2-connected. By Lemma 2.7, $G$ does not contain $C_{10}$ or $C_{8}$. By Lemma 2.10, $G$ contains an even cycle of length at most 6 . By definition, $G$ does not contain $C_{6}$. Hence $G$ contains $C_{4}$. Let $S$ be the vertex set of a 4-cycle in $G$, and let $R=V(G)-S$.

First suppose that $G[R]$ contains a 3-path $\langle u, z, v\rangle$. Since $G$ is 2-connected, two disjoint paths connect $\{u, z, v\}$ to $S$. Choosing shortest such paths, one has $u$ or $v$ as an endpoint, yielding $H_{2,3} \subseteq G$. This contradicts Lemma 2.6 ; we conclude $\Delta(G[R]) \leq 1$. 


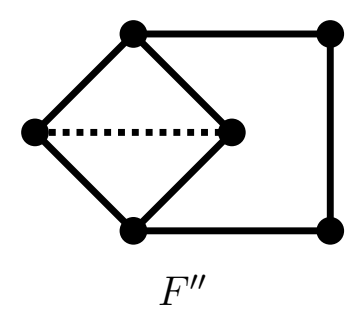

Figure 3: A forbidden subgraph for uniquely $C_{6}$-saturated graphs.

Suppose $u v \in E(G[R])$. Since $G$ is 2-connected and the only edges leaving $\{u, v\}$ go to $S$, there are distinct vertices $x, y \in S$ such that $\langle x, u, v, y\rangle$ is a 4 -path. Since $G$ cannot contain $C_{6}$, it contains the graph $F^{\prime \prime}$ in Figure 3. Since $F^{\prime \prime}$ has two nonadjacent vertices connected by more than one 6-path, $G$ is not uniquely $C_{6}$-saturated.

We may therefore assume that $R$ is an independent set. Since $G$ is 2-connected, each vertex in $R$ has at least two neighbors in $S$. If $|V(G)| \geq 6$, then let $u$ and $v$ be vertices in $R$. If two neighbors of each can be chosen in $S$ that are not the same pair, then $G[S \cup\{u, v\}]$ contains a 6-cycle or two 6-paths with endpoints $u$ and $v$, as shown in Figure 4. Hence $u$ and $v$ have degree 2 and have the same two neighbors in $S$. This makes them twins, which is forbidden by Lemma 2.9. We conclude that $G$ contains at most five vertices, which yields $G \in\left\{K_{4}, K_{5}\right\}$. We conclude that there are no nontrivial uniquely $C_{6}$-saturated graphs.
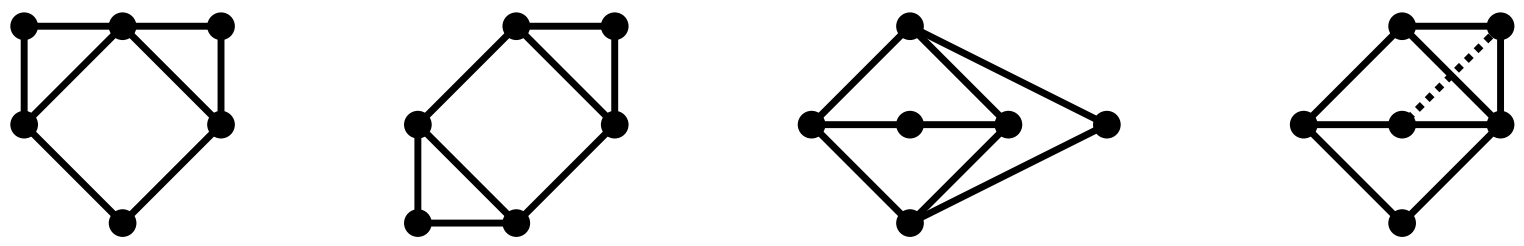

Figure 4: Forbidden subgraphs for uniquely $C_{6}$-saturated graphs.

The method for $C_{7}$ is similar.

Theorem 4.2. There are no nontrivial uniquely $C_{7}$-saturated graphs.

Proof. Let $G$ be a nontrivial uniquely $C_{7}$-saturated graph. By Corollary 2.5 we may assume that $G$ is 2-connected. By Lemma 2.7, $G$ does not contain $C_{12}$ or $C_{10}$. By Lemma 2.10, $G$ contains $C_{8}, C_{6}$, or $C_{4}$. Let $C$ be a longest cycle among the even cycles in $G$ with length at most 8, and let $R=V(G)-V(C)$. In each of several cases, we obtain a contradiction.

Case 1: $C$ has length 8. If $C$ has a chord joining vertices separated by distance 2 or 3 along $C$, then $G$ contains $C_{7}$ or $H_{2,4}$ and is not uniquely $C_{7}$-saturated. Hence any chord of $C$ joins opposite vertices on $C$. 
By Lemma 2.6, $H_{4,2} \nsubseteq \subseteq G$, so $R$ is an independent set. Because $G$ is 2-connected, each vertex in $R$ has at least two neighbors in $V(C)$. Consider $x \in R$. If $x$ has neighbors on $C$ that are not consecutive, then $G$ contains $H_{2,4}, C_{7}$, or $H_{3,3}$ and is not uniquely $C_{7}$-saturated. Hence every vertex of $R$ has exactly two neighbors on $C$, and they are consecutive on $C$.

Since twins are forbidden, two vertices of $R$ cannot be adjacent to the same consecutive pair. However, two vertices of $R$ adjacent to distinct consecutive pairs yield $C_{10}$ in $G$, which is forbidden. We conclude $|R| \leq 1$. If $|R|=1$ and $C$ has a (diametric) chord, then $H_{3,3} \subseteq G$, which is forbidden. If $|R|=1$ and $C$ has no chord, then adding any diametric chord completes no 7-cycle.

Hence we may assume that $V(G)=V(C)$ and $C$ has only diametric chords. Three diametric chords of an 8-cycle yield two 7-cycles (each omits one of the vertices not incident to a chord). Hence $G$ has at most one chord $e$. However, now no 7-path connects two vertices not adjacent to either endpoint of $e$.

Case 2: $C$ has length 6. By Lemma 2.6. $H_{3,3}$ is not a subgraph of $G$. Because $G$ is 2-connected, it follows that $G[R]$ has no component with at least three vertices. If $R$ is not independent, then there is a chordal path $P$ of length 3 connecting two vertices on $C$. If those vertices are consecutive or separated by distance 2 along $C$, then $G$ contains $C_{8}$ or $C_{7}$, which is forbidden. If $P$ joins opposite vertices on $C$, then two 7-paths join the neighbors on $C$ of one of the endpoints of $P$.

Hence $R$ is independent. Since $G$ is 2-connected, each vertex of $R$ has at least two neighbors in $V(C)$. Consecutive neighbors on $C$ yield $C_{7}$. Neighbors at distance 2 along $C$ yield two 7-paths with the same endpoints. Hence every vertex of $R$ is adjacent precisely to two opposite vertices on $C$. Now any two vertices of $R$ are twins or yield $C_{8}$, both forbidden.

If $R=\varnothing$, then $G=K_{6}$, so we may let $R=\{x\}$. The neighbors of $x$ are opposite vertices $y$ and $z$ on $C$. If $C$ has any non-diametric chord, then two 7-paths connect $x$ to some vertex on $C$. A diametric chord other than $y z$ creates $C_{7}$. Hence the only possible chord is $y z$. Now $\{y, z\}$ is a separating set in $G$ such that $G-\{y, z\}$ has three components, and the addition of a chord of $C$ incident to $y$ or $z$ cannot complete a spanning cycle in $G$.

Case 3: $C$ has length 4. Since $G$ is 2-connected, there is a chordal path joining two vertices of $C$. If $V(C)$ is a clique, then a chordal path of length 3,4 , or at least 5 creates copies of $C_{6}, C_{7}$, or $H_{2,4}$, respectively, all forbidden. Hence every chordal path has length 2. Since $|V(G)| \geq 7$, we conclude that $G$ contains $C_{6}$ or twins, both forbidden. We may therefore assume that $C$ is a 4-cycle whose chords are not both present. Let $u$ and $v$ be nonconsecutive on $C$ such that $u v \notin E(G)$, and let $x$ and $y$ be the other vertices of $C$. 
The 7-path $P$ with endpoints $u$ and $v$ also visits $x$ and $y$, since otherwise $C_{8} \subseteq G$, which was forbidden in Case 1 . Let $V(C)$ occur in the order $u, x, y, v$ along $C$. The path $P$ uses exactly three vertices of $R$. No matter how the three extra vertices are allocated to the three subpaths connecting vertices of $C$, a 6 -cycle is created in $G$, excluded by Case 2 .

We have also proved there are no uniquely $C_{8}$-saturated graphs. The proof uses the approach above, but more cases and details are needed to exclude the shorter even cycles. Hence we omit the proof.

\section{Finitely Many Uniquely $C_{t}$-saturated graphs}

In this section, we present the proof of Theorem 1.1 that for any $t \geq 6$ there are only finitely many uniquely $C_{t}$-saturated graphs. The main idea is to reduce the problem to the 2-connected case, showing that if there are finitely many uniquely $C_{t}$-saturated graphs that are 2-connected, then there are finitely many uniquely $C_{t}$-saturated graphs. For the first step, we restrict the ways that 2-connected uniquely $C_{t}$-saturated graphs can be combined.

Lemma 5.1. If $t \geq 6$ and $G$ is a 2-connected uniquely $C_{t}$-saturated graph, then no uniquely $C_{t}$-saturated graph $F$ has blocks $G^{\prime}$ and $G^{\prime \prime}$ isomorphic to $G$ such that $G^{\prime}$ and $G^{\prime \prime}$ share a cut-vertex of $F$ that can be viewed as the same vertex of $G$ in $G^{\prime}$ and $G^{\prime \prime}$.

Proof. Let $x$ be the vertex of $G$ in both $G^{\prime}$ and $G^{\prime \prime}$, no edge of $F$ joins $V\left(G^{\prime}-x\right)$ and $V\left(G^{\prime \prime}-x\right)$. For $y \in V(G-x)$, let $y^{\prime}$ and $y^{\prime \prime}$ be the corresponding vertices in $G^{\prime}$ and $G^{\prime \prime}$. If $G$ has distinct paths from $y$ to $x$ with lengths summing to $t-1$, then $F$ has two $t$-paths with endpoints $y^{\prime}$ and $y^{\prime \prime}$. Hence the unique $t$-path with endpoints $y^{\prime}$ and $y^{\prime \prime}$ consists of copies in $G^{\prime}$ and $G^{\prime \prime}$ of a unique $(t+1) / 2$-path $P_{y}$ in $G$ with endpoints $y$ and $x$. This uniqueness implies that $G$ is not complete (also $t$ is odd). We consider two cases.

Case 1: $x$ is adjacent to all of $V(G-x)$. Let $\hat{t}=(t-1) / 2$, so each $P_{y}$ is a $(\hat{t}+1)$-path. Since $P_{y}$ is unique and $x$ dominates $V(G-x)$, each $y \in V(G-x)$ starts exactly one $\hat{t}$-path in $G-x$; it is $P_{y}-x$. Let $z$ be the other endpoint of $P_{y}-x$. Vertex $y$ cannot have a neighbor in $G-x$ outside $P_{y}$, since $G$ would then have distinct paths from $z$ to $x$ with lengths $\hat{t}+1$ and $\hat{t}-1$ having sum $t-1$. Also $y$ cannot have a neighbor on $P_{y}$ other than its neighbor in $P_{y}$, since distinct $\hat{t}$-paths in $G-x$ would then start at $z$. Hence $d_{G-x}(y)=1$. With $y$ chosen arbitrarily, $G-x$ is 1-regular. Hence $2=\hat{t}=(t-1) / 2$, so this case requires $t=5$.

Case 2: $x$ has a nonneighbor $y$ in $G$. Since $G$ is not complete, by Lemma 2.10 $G$ has an even cycle $C$ of length at most $2 t-6$. Since $G$ is connected, there is a shortest path $Q$ 
connecting $x$ to the copy of $C$ in $G^{\prime}$. Since $x$ has a nonneighbor $y$ in $G$, there is a unique $t$-path $P$ in $G^{\prime \prime}$ with endpoints $x$ and $y^{\prime \prime}$. Letting $2 k$ be the length of $C$, the subgraph $C \cup Q \cup P$ of $F$ contains $H_{k, t-k-1}$, which is forbidden by Lemma 2.6.

Lemma 5.2. If there are finitely many 2-connected uniquely $C_{t}$-saturated graphs, then there are finitely many uniquely $C_{t}$-saturated graphs.

Proof. The diameter of a $C_{t}$-saturated graph is at most $t-1$. Hence the diameter of the

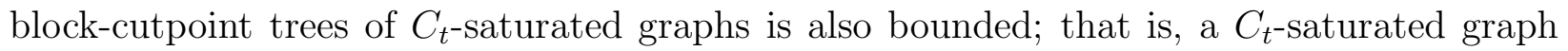
cannot contain a path that contains edges from more than $t-1$ blocks.

Every block of a uniquely $C_{t}$-saturated graph is uniquely $C_{t}$-saturated. With finitely many 2-connected uniquely $C_{t}$-saturated graphs, the number of vertices in any single block of a uniquely $C_{t}$-saturated graph is bounded. If there are infinitely many uniquely $C_{t^{-} \text {-saturated }}$ graphs, then they must exist with arbitrarily many blocks. With bounded diameter in the block-cutpoint tree, they must have block-cutpoint trees with arbitrarily many leaves.

Since the distance between leaves is bounded, there must be arbitrarily many leaf blocks having a common cutvertex. Since the number of possible leaf blocks is bounded, there must exist instances with arbitrarily many isomorphic leaf blocks having a common cut-vertex. Since the number of vertices in the blocks are bounded, there must be instance with two isomorphic leaf blocks sharing a cut-vertex that has the same identity in each of the two blocks. This contradicts Lemma 5.1.

To complete the proof of the theorem, we need to show that there are finitely many 2-connected uniquely $C_{t}$-saturated graphs. We do this by bounding the number of vertices in such a graph. Two lemmas are needed.

The first extends Lemma 2.9. When $G$ has twins, it has an automorphism exchanging the twins but leaving all other vertices fixed. The twins are components of $G-S$, where $S$ is their common neighborhood. We next consider a situation in which $G-S$ contains four isomorphic components. Given a set $S \subseteq V(G)$ and $v \in V(G)-S$, let a $v, S$-path be a path connecting $v$ to a vertex in $S$ with no internal vertices in $S \cup\{v\}$.

Lemma 5.3. Given $t \geq 8$, let $G$ be a 2-connected graph with $S \subset V(G)$. Suppose that $G-S$ has distinct isomorphic components $F_{1}, F_{2}, F_{3}$ and $F_{4}$ such that for all $i \in\{2,3,4\}$ there is an automorphism $\varphi_{i}$ of $G$ such that (1) $\varphi_{i}^{2}$ is the identity, (2) $\varphi_{i}\left(F_{1}\right)=F_{i}$, and (3) $\varphi_{i}$ fixes all vertices outside $F_{1} \cup F_{i}$. If $G$ is uniquely $C_{t}$-saturated, then every vertex of $F_{1}$ that has a neighbor in $S$ starts some path in $F_{1}$ with length $t-2$. 
Proof. Let $\hat{t}=(t-1) / 2$. We first prove that for every $x_{1} \in V\left(F_{1}\right)$ there is an $x_{1}, S$-path of length $\hat{t}$. If this fails for some $x_{1} \in V\left(F_{1}\right)$, then let $x_{2}=\varphi_{2}\left(x_{1}\right)$. Since $G$ is uniquely

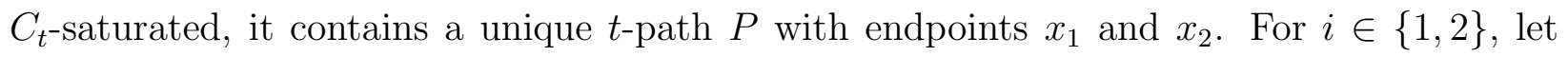
$P^{i}$ be the $x_{i}, S$-path contained in $P$. If $P^{1}$ and $P^{2}$ have the same endpoint in $S$, then they have different lengths, since $G$ has no $x_{1}, S$-path of length $\hat{t}$. Otherwise, they have different endpoints in $S$. In both cases, a second $t$-path with endpoints $x_{1}$ and $x_{2}$ consists of $P$ with each $P^{i}$ replaced by $\varphi_{2}\left(P^{3-i}\right)$. This contradiction proves the claim.

Hence for all $x \in F_{1}$ there is an $x, S$-path of length $\hat{t}$. Let $x$ be a vertex of $F_{1}$ having a neighbor $y \in S$. Suppose that $x$ starts no path in $F_{1}$ with length $t-2$. Since $G$ contains an $x, S$-path $P$ of length $\hat{t}$, we may choose $z \in V\left(F_{1}\right)$ at distance $\hat{t}-1$ from $x$ along $P$. Let $P^{\prime}$ be the $\hat{t}$-path from $x$ to $z$ along $P$. Because $G$ is 2-connected, $G-y$ has a shortest path connecting $V\left(P^{\prime}\right)$ and $S$; call it $Q^{\prime}$, with endpoints $x^{\prime} \in V\left(P^{\prime}\right)$ and $y^{\prime} \in S$. Let $Q$ be the path from $y$ to $y^{\prime}$ consisting of the edge $y x$, the subpath of $P^{\prime}$ from $x$ to $x^{\prime}$, and $Q^{\prime}$ (see Figure 5 ). Let $k$ be the length of $Q$. Since $x$ starts no path of length $t-2$ in $F_{1}$, we have $k \leq t-1$.

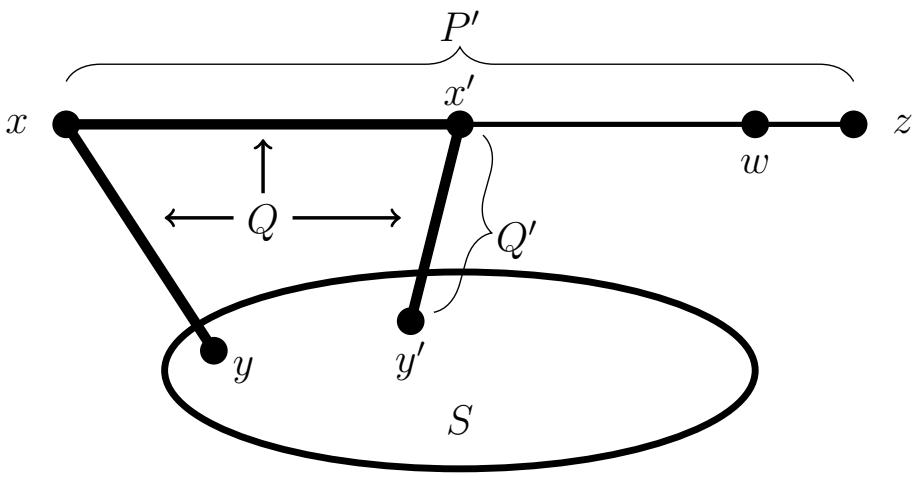

Figure 5: Paths in $F_{1}$. The bold path is $Q$, which overlaps with a portion of $P^{\prime}$.

Note that $\varphi_{2}(Q) \cup \varphi_{3}(Q)$ is a cycle $C$ of length $2 k$ in $G$, and the union of $\langle y, x\rangle$ with $P^{\prime}$ is a path of length $\hat{t}$. If $k \geq \hat{t}$, then $C \cup\langle y, x\rangle \cup P^{\prime}$ contains $H_{k, t-k-1}$.

Hence we may assume $k<\hat{t}$. The portion of $Q$ along $P^{\prime}$ has length at most $k-2$. Hence the path from $y^{\prime}$ to $z$ in $Q^{\prime} \cup P^{\prime}$ has length at least $\hat{t}-k+2$. Let $\hat{P}$ be its subpath of length $\hat{t}-k$ starting from $y^{\prime}$, and let $w$ be the other endpoint of $\hat{P}$. For $i \in\{2,3\}$, the concatenation of $P^{\prime}, x y, \varphi_{i}(Q)$, and $\varphi_{4}(\hat{P})$ has $\hat{t}+1+k+\hat{t}-k$ vertices; hence it is a $t$-path with endpoints $z$ and $\varphi_{4}(w)$. Again this contradicts $G$ being uniquely $C_{t}$-saturated, so $x$ must start a path in $F_{1}$ with length $t-2$.

Lemma 5.4. There are finitely many 2-connected uniquely $C_{t}$-saturated graphs. 
Proof. By Theorems 4.1 and 4.2 , there are no uniquely $C_{6}$-saturated or $C_{7}$-saturated graphs. Hence we may assume $t \geq 8$. It suffices to prove that the number of vertices in a 2 connected uniquely $C_{t}$-saturated graph $G$ is bounded. In order to prove this, we prove that the maximum degree in such a graph is bounded. Since the diameter of a uniquely $C_{t}$-saturated graph is less than $t$, this bounds the number of vertices.

By Lemma 2.10, $G$ contains an even cycle $C$ of length at most $2 t-6$. Let $C$ have length $2 k$, and let $S=V(C)$. By Lemma 2.6, $G$ does not contain $H_{k, t-k-1}$, and hence all paths leaving $S$ have length at most $t-k-2$.

Let $R_{t-k-i}$ be the set of vertices $v$ outside $S$ such that longest $v, S$-paths have length $i$. Because $G$ is connected and $H_{k, t-k-1} \nsubseteq \subseteq G$, it follows that every vertex of $G-S$ lies in $R_{t-k-i}$ for some $i$ with $2 \leq i \leq t-k-1$. Also set $R_{0}=S$; this is $R_{t-k-i}$ for $i=t-k$. We proceed by induction on $i$ to prove the existence of $c_{i}$ such that $d_{G}(v) \leq c_{i}$ when $v \in R_{t-k-i}$ and $2 \leq i \leq t-k$.

For $v \in R_{t-k-2}$, the neighbors of $v$ lie in $S$ or on a $v, S$-path of length $t-k-2$, since $H_{k, t-k-1} \notin G$. Thus $d_{G}(v) \leq t+k-3 \leq 2 t-6$, and we can set $c_{2}=2 t-6$.

Now consider $v \in R_{t-k-i}$, where $3 \leq i \leq t-k$. Let $P$ be a $v, S$-path of length $t-k-i$, and let $S^{\prime}=S \cup V(P)$. Let $N^{\prime}(v)=N(v)-S^{\prime}$. By Lemma 2.9, $G$ does not contain twins, so at most $2^{t+k-i-1}$ vertices in $N^{\prime}(v)$ have neighborhoods contained in $S^{\prime}$. Let $N^{\prime \prime}(v)$ be the set of vertices in $N^{\prime}(v)$ having a neighbor outside $S^{\prime}$, so $\left|N^{\prime \prime}(v)\right| \geq d_{G}(v)-(t+k-i-1)-2^{t+k-i-1}$.

If any component of $G-S^{\prime}$ contains an $(i-1)$-path starting at a vertex of $N^{\prime \prime}(v)$, then $G$ contains $H_{k, t-k-1}$ and is not uniquely $C_{t}$-saturated. Let $F$ be the subgraph of $G-S^{\prime}$ that consists of the components of $G-S^{\prime}$ that contain vertices in $N^{\prime \prime}(v)$. Hence each vertex of $F$ lies in $R_{t-k-j}$ for some $j$ with $2 \leq j<i$. By the induction hypothesis, $F$ has maximum degree bounded by $\max _{j<i} c_{j}$. With also bounded diameter, the number of vertices in a component of $F$ is bounded by some value $h(t)$.

Let $F^{\prime}$ be a possible component of $F$. For each component of $F$ isomorphic to $F^{\prime}$, we can list the neighborhood in it of each vertex of $S^{\prime}$; there are $\left(2^{\left|V\left(F^{\prime}\right)\right|}\right)^{\left|S^{\prime}\right|}$ possible such lists. If $F$ has more than $3 \cdot 2^{\left|V\left(F^{\prime}\right)\right| \cdot\left|S^{\prime}\right|}$ components isomorphic to $F^{\prime}$, then some four of them yield the same list. Each vertex of $S^{\prime}$ has the same neighborhood in these four components, so together with $S^{\prime}$ they satisfy the conditions of Lemma 5.3. The resulting path with length $t-2$ from a vertex of $N^{\prime \prime}(v)$ would contradict $G$ being uniquely $C_{t}$-saturated.

Since $\left|S^{\prime}\right|=t+k-i<2 t$, we have at most $3 \cdot 2^{2 t \cdot h(t)}$ components of $F$ isomorphic to $F^{\prime}$, and there are fewer than $2^{\left(\begin{array}{c}(t) \\ 2\end{array}\right)}$ isomorphism classes of graphs with at most $h(t)$ vertices. Hence we have a bound (in terms of $t$ ) on $\left|N^{\prime \prime}(v)\right|$ and hence also a bound $c_{i}$ on $d_{G}(v)$. 
Since every vertex of $G$ lies in $R_{t-k-i}$ for some $i$ with $2 \leq i \leq t-k$, we have established $\max _{2 \leq i \leq t-k} c_{i}$ as a bound on the degrees of all vertices in $G$.

Lemmas 5.2 and 5.4 complete the proof of Theorem 1.1 there are finitely many uniquely $C_{t}$-saturated graphs.

\section{References}

[1] L.W. Berman, G.E. Chappell, J.R. Faudree, J. Gimbel, and C. Hartman, Uniquely treesaturated graphs, preprint.

[2] J. Cooper, personal communication (2011).

[3] J. Cooper, J. Lenz, T.D. LeSaulnier, P. Wenger, and D.B. West, Uniquely $C_{4}$-saturated graphs, Graphs and Comb. 28 (2012), no. 2, 189-197.

[4] P. Erdős, A. Hajnal and J.W. Moon, A problem in graph theory, Amer. Math. Monthly 71 (1964), 1107-1110.

[5] P. Erdős, A. Rényi, and V. Sós, On a problem of graph theory, Studia Sci. Math. Hungar. 1 (1966), 215-235.

[6] S. Hartke and D. Stolee, Uniquely $K_{r}$-saturated graphs, Electron. J. Combin. 19 (2012), no. 4, \#P6.

[7] W. Mantel, Problem 28, soln. by H. Gouwentak, W. Mantel, J. Teixeira de Mattes, F. Schuh and W.A. Wythoff, Wiskundige Opgaven 10 (1907) 60-61.

[8] P. Turán, Eine Extremalaufgabe aus der Graphentheorie, Mat. Fiz. Lapok 48 (1941), $436-452$. 